\title{
Resolving the atmospheric octant by an improved measurement of the reactor angle
}

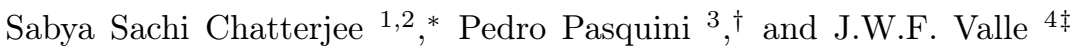 \\ 1 Institute of Physics, Sachivalaya Marg, Sainik School Post, Bhubaneswar 751005, India \\ 2 Homi Bhabha National Institute, Training School Complex, Anushakti Nagar, Mumbai 400085, India \\ ${ }^{3}$ Instituto de Física Gleb Wataghin - UNICAMP, 13083-859, Campinas SP, Brazil \\ 4 AHEP Group, Institut de Física Corpuscular - C.S.I.C./Universitat de València, Parc Cientific de Paterna. \\ C/Catedratico José Beltrán, 2 E-46980 Paterna (València) - SPAIN
}

\begin{abstract}
Taking into account the current global information on neutrino oscillation parameters we forecast the capabilities of future long baseline experiments such as DUNE and T2HK in settling the atmospheric octant puzzle. We find that a good measurement of the reactor angle $\theta_{13}$ plays a key role in fixing the octant of the atmospheric angle $\theta_{23}$ with such future accelerator neutrino studies.
\end{abstract}

PACS numbers: 12.15.-y,13.15.+g,14.60.Pq,14.60.St

\section{INTRODUCTION}

The discovery of neutrino oscillations as a result of solar and atmospheric studies constitutes a major milestone in astroparticle physics [1, 2]. Earthbound experiments based at reactors and accelerators have not only provided a confirmation of the oscillation picture but also brought the field into the precision age. Despite the great experimental effort, however, two of the oscillation parameters remain poorly determined, namely the atmospheric mixing angle $\theta_{23}$ and the $\mathrm{CP}$ violating phase $\delta_{C P}[3.5]$.

Underpinning these parameters as well as determining the neutrino mass ordering constitute important challenges in the agenda of upcoming oscillation experiments, needed to establish the three-neutrino paradigm. Concerning the two poorly known neutrino parameters, $\theta_{23}$ yields two degenerate solutions [3] 5 , well known as the octant problem [6]. One of them is known as lower octant (LO): $\theta_{23}<\pi / 4$ while the other is termed as higher octant (HO): $\theta_{23}>\pi / 4$. The role of $\theta_{13}$ and its precise determination has been stressed in early papers [7, 8]. The actual discovery of large $\theta_{13}$ that has opened a tremendous opportunity for the long-baseline neutrino oscillation experiments to resolve the octant issue within the standard 3-flavor framework. This may however be just an approximation to the true scenario, which may involve new physics such as non-unitarity [9] which may have important effects on the propagation of astrophysical neutrinos [13, 14, non-standard interactions [15] as well as a light sterile neutrino [16].

\footnotetext{
* sabya@iopb.res.in

$\dagger$ pasquini@ifi.unicamp.br

$\ddagger$ valle@ific.uv.es, URL: http://astroparticles.es/
}

Recently there have been many papers addressing the octant issue within the standard $3 \nu$ scenario $17-23$. However it has also been shown recently that the octant sensitivity may completely change in the presence of the above non-standard features, i.e. non-unitarity [24], nonstandard interaction [25] or a light sterile neutrino [26].

In this letter we specifically focus on the reactor angle $\theta_{13}$ and on whether an improved precision in its measurement from reactors, combined with results from future long baseline experiments such as DUNE and/or T2HK, could provide a final resolution to the octant puzzle. Taking into account current global neutrino oscillation parameter fits, we forecast the potential of DUNE [27] and T2HK [28] for pinning down the correct octant of $\theta_{23}$. We find that a sufficiently good measurement of the reactor angle $\theta_{13}$ directly fixes values of $\theta_{23}$ for which the octant of the atmospheric angle can be distinguished.

\section{THEORY PRELIMINARIES}

Following [29], the appearance and survival oscillation probabilities in the presence of matter can be written approximately as

$$
\begin{aligned}
P_{\mu e} \approx & 4 s_{13}^{2} s_{23}^{2} \sin ^{2} \Delta_{31} \\
& +2 \alpha \Delta_{31} s_{13} \sin 2 \theta_{12} \sin 2 \theta_{23} \cos \left(\Delta_{31} \pm \delta_{C P}\right) \\
= & P_{0}+P_{I} \\
P_{\mu \mu} \approx & 1-\sin ^{2} 2 \theta_{23} \sin ^{2} \Delta_{31}-4 s_{13}^{2} s_{23}^{2} \frac{\sin ^{2}(A-1) \Delta_{31}}{(A-1)^{2}}
\end{aligned}
$$

where $s_{i j}=\sin \theta_{i j}, \alpha=\frac{\Delta m_{21}^{2}}{\Delta m_{31}^{2}}, \Delta_{31}=\frac{\Delta m_{31}^{2} L}{4 E}$ and the function $\mathrm{A}=\frac{2 E V_{C C}}{\Delta m_{31}^{2}}$ describes the matter profile. Here 
$V_{C C}$ is the charged current potential in earth matter, while $\mathrm{L}$ and $\mathrm{E}$ are the propagation distance and energy of the neutrinos, respectively. The \pm sign in front of $\delta_{\mathrm{CP}}$ corresponds to neutrinos (upper sign) and antineutrinos (lower sign). The term $P_{0}$ is the octant sensitive term, whereas the term related to $\sin ^{2} 2 \theta_{23}$ generates the octant degeneracy.

An experiment is octant sensitive, if there is always a finite difference between the two probabilities corresponding to the two octants, despite the minimization performed over the different oscillation parameters. Mathematically,

$$
\Delta P \equiv P_{\mu e}^{\mathrm{HO}}-P_{\mu e}^{\mathrm{LO}} \neq 0
$$

Note that we assume that one of the two octants is the true octant in order to generate the data, while the other one is the false octant in order to simulate the theoretical model predictions. We will always assume that $\theta_{13}$ lies in its true value $\left(\sin ^{2} \theta_{13}=0.0234\right)$ in the true octant. Following Eq. 1, we can write

$$
\Delta P=\Delta P_{0}+\Delta P_{I} .
$$

Now, by expanding Eq. 1 around $\theta_{23}=\pi / 4 \pm \eta$ and $\sin ^{2} \theta_{13}=(1+\epsilon) \sin ^{2} \theta_{13}$ we get,

$$
P_{0}=(1 \pm 2 \eta+\epsilon) P^{\prime}+O(\epsilon \eta)
$$

where $\epsilon= \pm \delta\left(\sin ^{2} \theta_{13}\right)$ denotes the error on $\sin ^{2} \theta_{13}$ and the \pm sign in front of $\eta$ refers to HO (upper sign) and LO (lower sign) and,

$$
P^{\prime} \equiv P^{\prime}\left(\theta_{23}=\pi / 4, \theta_{13}=\theta_{13}^{\text {true }}\right)=2 s_{13}^{2} \sin ^{2} \Delta_{31} .
$$

leading to

$$
\Delta P_{0}=\left(P_{0}^{\mathrm{HO}}-P_{0}^{\mathrm{LO}}\right)=P^{\prime}(4 \eta \pm \epsilon) .
$$

The double sign in front of $\epsilon$ refers to the $\mathrm{LO}^{\text {true }}$ (upper sign) and $\mathrm{HO}^{\text {true }}$ (lower sign).

In the same manner, we can also write

$$
\begin{aligned}
\Delta P_{I}=B\left[\sin \theta_{13}^{\mathrm{HO}}\right. & \cos \left(\Delta_{31} \pm \delta_{\mathrm{CP}}^{\mathrm{HO}}\right) \\
& \left.\quad-\sin \theta_{13}^{\mathrm{LO}} \cos \left(\Delta_{31} \pm \delta_{\mathrm{CP}}^{\mathrm{LO}}\right)\right]
\end{aligned}
$$

where, $\mathrm{B}=4 \sin \theta_{12} \cos \theta_{12}(\alpha \Delta) \sin \Delta_{31}$. Notice that, as mentioned above, $\sin \theta_{13}^{\mathrm{HO}}$ and $\sin \theta_{13}^{\mathrm{LO}}$ change shape depending on true versus wrong octant.

For the time being suppose one neglects the error on $\sin ^{2} \theta_{13}$ by taking $\epsilon \rightarrow 0 . \Delta P_{0}$ is positive definite, while
$\Delta P_{I}$ can have either sign due to the presence of the unknown $\delta_{\mathrm{CP}}$. As a result $\Delta P$ may become zero for the unfavorable combinations of octant and $\delta_{C P}$, so that octant sensitivity can be completely lost. However, it has been noticed in the literature [17, 30] that this type of degeneracy can be lifted by using both neutrino and antineutrino channels and one can achieve good octant sensitivity in the 3-flavor scenario.

In the presence of a nonzero error on $\sin ^{2} \theta_{13}$, then $\Delta P_{0}$ is also a positive definite quantity, since the current error on $\sin ^{2} \theta_{23}$ is bigger than the error on $\sin ^{2} \theta_{13}$, i.e., we can safely assume $\eta>\epsilon$. In this case it is clear from Eqs. 5 and 6 that the unfavorable contribution coming from $\epsilon \neq 0$ changes the magnitude of $\Delta P_{0}$ and $\Delta P_{I}$ in such a way that overall value of $\Delta P$ decreases further than in the previous case. As a result the octant discrimination sensitivity decreases significantly even in the presence of neutrino and antineutrino channels. The larger the error, the less will be the resulting octant sensitivity. This will be clearly seen in the next section.

\section{SIMULATION DETAILS}

Here we present in some detail the experimental configurations of the DUNE and T2HK experiments used for this work. For a more detailed discussion see 31

DUNE: Deep Underground Neutrino Experiment (DUNE) is a long-baseline $(1300 \mathrm{~km})$ accelerator-based experiment with neutrinos travelling from Fermilab to South Dakota. Following the DUNE CDR [27, we are using a $40 \mathrm{Kt}$ LArTPC as its far detector, and a $80 \mathrm{GeV}$ proton beam with beam power $1.07 \mathrm{MW}$. A total 300 Kt.MW.yrs of exposure has been assumed with neutrino mode running for $3.5 \mathrm{yrs}$, and the antineutrino mode running for 3.5 yrs. All the signal and background event numbers have been matched following Table 3.5 and Table 3.6 of [27]. With this all the reconstructed neutrino and antineutrino energy spectra and sensitivity results have been reproduced as close as possible to the same reference. As a simplified case for all the neutrino and antineutrino appearance and disappearance channels we have assumed an uncorrelated $4 \%$ signal normalization error and $10 \%$ background normalization error.

T2HK : T2HK (Tokai to Hyper-Kamiokande) is an offaxis accelerator based experiment with baseline $295 \mathrm{~km}$. According to [28], it plans to use the same $30 \mathrm{GeV}$ proton beam as T2K, provided by the J-PARC facility and a 560 Kton (fiducial volume) Water Chernkov far detector. 
An integrated beam of power $7.5 \mathrm{MW} \times 10^{7} \mathrm{sec}$ has been assumed for this work which corresponds to $1.56 \times 10^{22}$ protons on target. In order to make the expected event numbers nearly the same for neutrinos and antineutrinos, we consider a 2.5 yrs of neutrino running mode and 7.5 yrs of antineutrino running mode. All the signal and background event numbers have been matched following Table 7 and Table 8 of Ref. 28] and all other sensitivity results have been reproduced with good agreement. As a simplified case we have assumed an uncorrelated $5 \%$ signal normalization error, and $10 \%$ background normalization error with no energy calibration error.

We have performed a realistic simulation by using the GLoBES package [32, 33]. The best fit values of the oscillation parameters were taken from [5] except for the top plot of Fig. 1, in which we have followed [3]. They are the following: $\sin ^{2} \theta_{12}=0.306, \sin ^{2} \theta_{13}=0.0216, \sin ^{2} \theta_{23}=$ 0.441 for $\mathrm{NH}, \delta_{C P}=1.45 \pi, \Delta m_{21}^{2}=7.5 \times 10^{-5} \mathrm{eV}^{2}$, and $\Delta m_{31}^{2}=2.524 \times 10^{-3}\left(-2.514 \times 10^{-3}\right) \mathrm{eV}^{2}$ for $\mathrm{NH}(\mathrm{IH})$. Here NH (IH) is short for normal hierarchy (inverted hierarchy). In all of our numerical analysis, we have assumed $\mathrm{NH}$ as fixed both in data and theory. In order to determine the sensitivity towards the measurement of the octant of $\theta_{23}$, we have defined the $\chi^{2}$ function as,

$$
\chi^{2}=\chi_{\mathrm{GLoBES}}^{2}+\chi_{\text {Priors }}^{2}
$$

where $\chi_{\text {GLoBES }}^{2}$ is the standard GLoBES Poissionian chisquared, while $\chi_{\text {Priors }}^{2}$ is given by,

$$
\chi_{\text {Priors }}^{2}=\sum_{i=2,3}\left(\frac{s_{1 i}^{2, \text { TRUE }}-s_{1 i}^{2, \text { TEST }}}{\delta\left(s_{1 i}^{2, \text { TRUE }}\right)}\right)^{2}
$$

with $\delta\left(s_{i j}^{2, \text { TRUE }}\right)$ is the $\sin ^{2} \theta_{i j}^{\mathrm{TRUE}}$ error from [5], while $s_{i j}^{2, \mathrm{~A}}=\sin ^{2} \theta_{i j}^{\mathrm{A}}$. Here $A=$ TRUE, TEST denote the true and test values of the angles respectively. We have not included either $\delta_{\mathrm{CP}}$ or $\theta_{23}$ priors, as we are focusing on the capability of each experiment to measure them. In order to distinguish the true octant from the false one. We define the chi-squared difference as $\Delta \chi_{\text {oct }}^{2}=\left|\chi_{\min }^{2}\left(\theta_{23} \leq \pi / 4\right)-\chi_{\min }^{2}\left(\theta_{23}>\pi / 4\right)\right|$. Here $\chi_{\text {min }}^{2}\left(\theta_{23}\right)$ is the $\chi^{2}$ function minimized over other oscillation parameters. Note that one can assume one of the octants (say, $\theta_{23} \leq \pi / 4$ ) as true and the other one as false, and vice-versa.

Recent reactor experiments have reached a precision at the percent level for the measurement of the reactor angle, fixing its central value around $\sin ^{2} \theta_{13} \sim 0.02$. Current and foreseen precision levels on the reactor angle are given in table I. For the simulation we took the central value of $\sin ^{2} \theta_{13}$ from the global fit [5], and vary the error on $\sin ^{2} \theta_{13}$ as a prior as in Eq. 8. This makes the analysis more robust, as taking a single experiment error cannot give a general picture for the value of $\sin ^{2} \theta_{13}$.

\begin{tabular}{ccccc}
\hline \hline & DC [34 & RENO [35] & Daya-Bay [36 & Global [5] \\
\hline \hline$s_{13}^{2} / 10^{-2}$ & 2.85 & 2.09 & 2.09 & 2.34 \\
$\delta\left(s_{13}^{2}\right) / s_{13}^{2}$ & $16.7 \%$ & $13.4 \%$ & $4.9 \%$ & $3.5 \%$ \\
$\delta\left(s_{13}^{2, \text { Expec }}\right) / s_{13}^{2}$ & $10 \%$ & $5 \%$ & $3.6 \%$ & $<3 \%$ \\
\hline
\end{tabular}

TABLE I. Current and expected values of the reactor mixing angle $\theta_{13}$ and its sensitivity for different experiments and current global neutrino oscillation fit. The expected values are based on [37]. The $\%$ is calculated by taking the $1 \sigma$ region from the central value.

\section{RESULTS AND DISCUSSION}

In this section we present our numerical results and their comprehensive discussion. In our whole analysis we have used a line-averaged constant matter density of 2.95 $\mathrm{gm} / \mathrm{cm}^{3}$ for DUNE and $2.8 \mathrm{gm} / \mathrm{cm}^{3}$ for T2HK within the PREM [38, 39] profile.

\section{A. Precision measurement}

Fig. 1 represents the $4 \sigma$ confidence level measurement of $\theta_{23}$ and $\delta_{\mathrm{CP}}$ for various combinations of the relative error associated with $\sin ^{2} \theta_{13}$. The symbol "star" in the body of the plot corresponds to the best fit value for two assumptions: (I) Top, the global fit in [3] and (II) Bottom, the global fit in [5]. The left (right) panel is for DUNE (T2HK). The cyan band corresponds to $1.2 \%$, the orange band corresponds to $2.4 \%$, the red band is for $3.6 \%$ and the blue band corresponds to $4.8 \%$ error on $\sin ^{2} \theta_{13}$. In contrast, the green band is generated by the free marginalization over $\sin ^{2} \theta_{13}$. We have marginalized over $\Delta m_{31}^{2}$ and $\theta_{12}$ with $1 \sigma$ prior on $\sin ^{2} \theta_{12}$ taken from [5]. Left panel, clearly shows that DUNE can not exclude the wrong octant for errors above $\sim 2.0 \%$, while it can surely exclude the wrong octant at $4 \sigma$ confidence if $\sin ^{2} \theta_{13}$ is more tightly constrained as for the case of option (II), as seen from the cyan shaded region. Thanks to its higher statistics in the disappearance channel, T2HK performs better, and can exclude the wrong octant up to $2.4 \% \sin ^{2} \theta_{13}$ error for the option (I), and $3.6 \%$ in the 

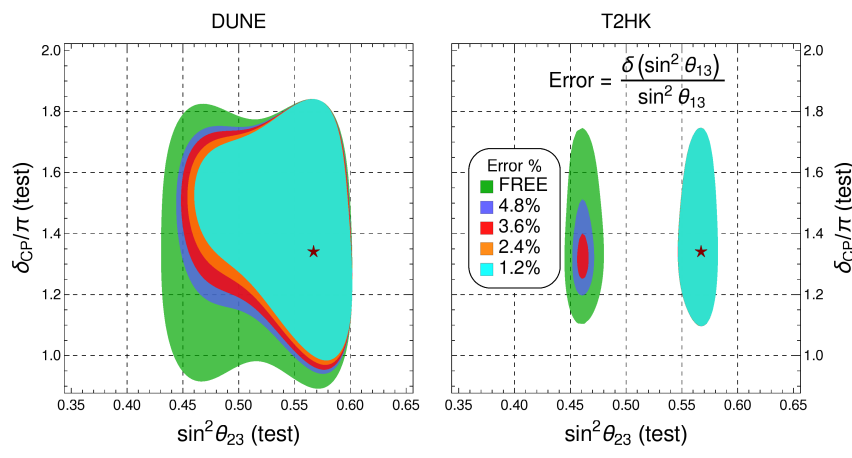

DUNE
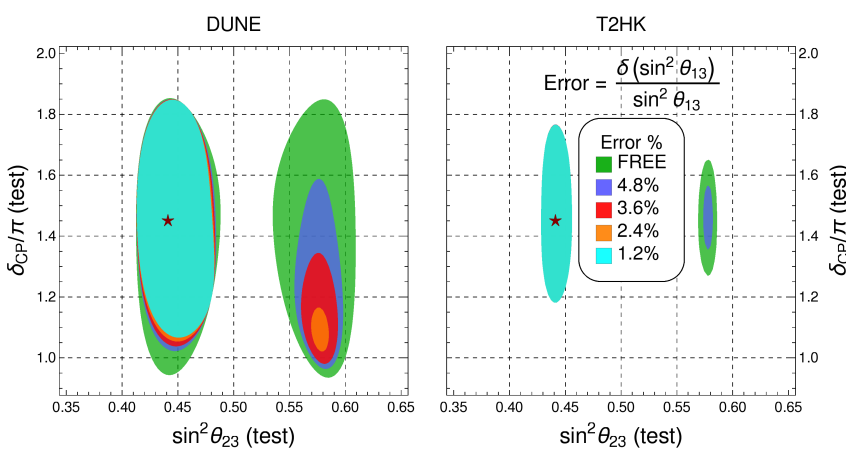

FIG. 1. Precision measurement of $\theta_{23}$ and $\delta_{C P}$ at $4 \sigma\left(\Delta \chi^{2}=\right.$ 19.33) confidence. Left (Right) panels correspond to DUNE (T2HK). Differently shaded (colored) regions correspond to various errors associated with $\sin ^{2} \theta_{13}$. The Top panel uses the Global Fit in [3], while the Bottom panel corresponds to the Global Fit in [5]. The star denotes the bestfit point.

case of the option (II), i.e., T2HK can measure the atmospheric mixing angle very precisely.
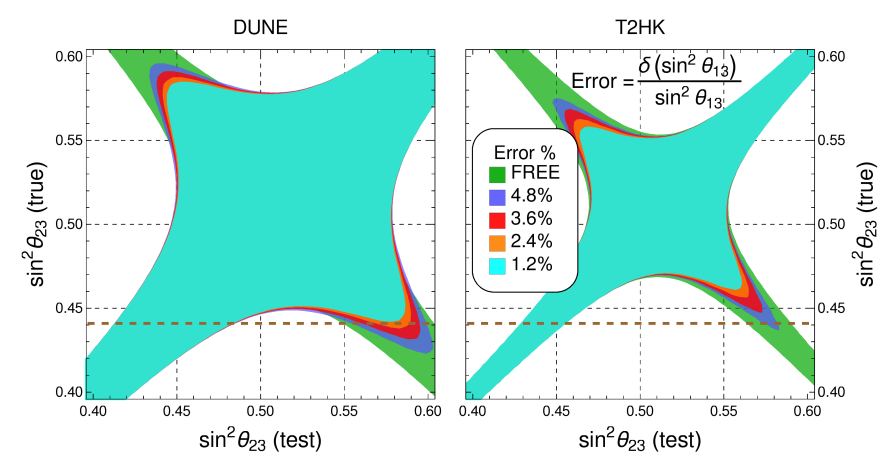

FIG. 2. $4 \sigma\left(\Delta \chi^{2}=19.33\right)$ precision measurement of $\theta_{23}$. The left (right) panel is for DUNE (T2HK). Differently shaded regions correspond to various errors associated with $\sin ^{2} \theta_{13}$. The thick dashed line represents the current best fit value from 3 .

Note that from Fig. 1 one can compare the result for two oscillation fits. Although Ref. [5] contains the most recent data from Daya-Bay, $\mathrm{T} 2 \mathrm{~K}$, and $\mathrm{NO} \nu \mathrm{A}$, which con- strain $\theta_{13}$, they do not include the atmospheric data sample, as in 3]. While the latter has an impact upon which is the preferred octant, it has worse precision on the $\sin ^{2} \theta_{13}$, which plays a role in the octant discrimination. The two analyses are therefore complementary, though an update of [3] is clearly desirable (work in this direction is currently underway). Our work shows that, in both cases, DUNE by itself will not be enough to solve the octant problem, while T2HK can have a better chance to uncover the right value of the atmospheric angle. In contrast to the determination of the neutrino mass ordering, despite the unprecedented level of precision on $\theta_{13}$ that may be reached in future studies, there will always be a region that is octant blind in any experiment, close to the maximality limit.

We now turn to a very general question, namely, how well can these two experiments measure $\theta_{23}$ irrespective of its true value chosen by nature.

Fig. 2 addresses this issue. The simulation procedure is exactly the same as for fig. 1. except for the fact that we have marginalized over $\delta_{\mathrm{CP}}$ both in the data and the theory. As a result this figure represents the most conservative scenario. If we draw a horizontal line for each true value of $\sin ^{2} \theta_{23}$, it touches the different colored shaded regions associated to different $\sin ^{2} \theta_{13}$ errors. The horizontal boundary of each touched shaded region corresponding to a particular color represents the $4 \sigma$ uncertainty on $\sin ^{2} \theta_{23}$. It can be determined simply by looking at the brown thick dashed line at $\sin ^{2} \theta_{23}$ (true) $=0.441$ and focusing on the cyan band. This procedure extracts all the relevant information coming from fig. 1. It is noticeable that DUNE measures the LO $\left(\sin ^{2} \theta_{23}\right.$ (true $)<$ $0.45)$ better than the HO. However, the performance of T2HK is substantially higher than that of DUNE in both the octants. An important consequence of the green area in Fig. 2 is the fact that neither DUNE nor T2HK can distinguish the octant without prior knowledge of $\theta_{13}$.

\section{B. Octant discrimination}

Here we analyse the potential of DUNE and T2HK for excluding the wrong octant provided the data is generated in the true octant. Fig. 3 illustrates the octant sensitivity as a function of the relative error on $\sin ^{2} \theta_{13}$. The left (right) panel corresponds to the result for DUNE (T2HK). The colored curves indicate the sensitivity for discriminating the false octant from the true one depending on the relative $\sin ^{2} \theta_{13}$ error. The red, green, blue 

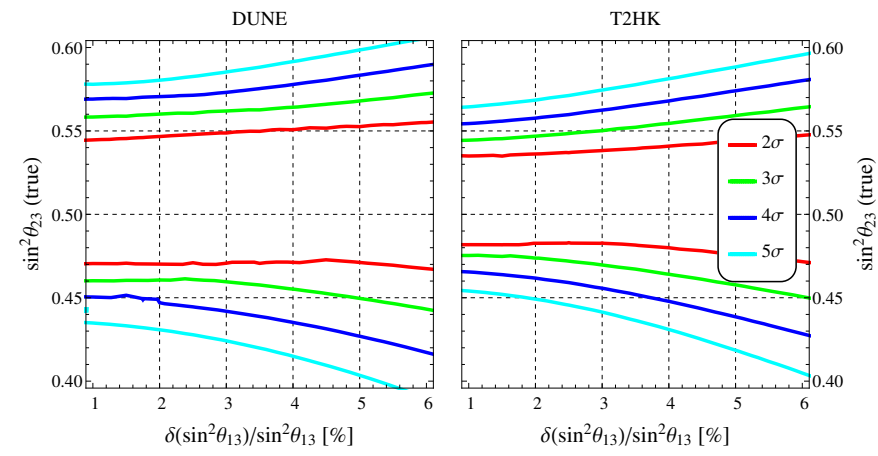

FIG. 3. Octant discrimination potential as a function of the relative error on $\sin ^{2} \theta_{13}$ for the true value of $\delta_{\mathrm{CP}}^{\mathrm{TRU}}=$ $1.45 \pi$. The left (right) panel represents the results for DUNE (T2HK). The red, green, blue and cyan curves delimit the $\theta_{23}$ "octant-blind" region corresponding to $2,3,4$ and $5 \sigma\left(\Delta \chi^{2}=\right.$ $4,9,16$, and 25 respectively) confidence.

and cyan correspond to the $2 \sigma, 3 \sigma, 4 \sigma$ and $5 \sigma$ confidence level cases, respectively. NH is assumed as the true hierarchy both in data and theory (note that the IH case can be considered in exactly the same way). Concerning theory, we have marginalized over the oscillation parameters $\theta_{12}, \theta_{13}, \theta_{23}, \delta_{\mathrm{CP}}$ and $\Delta m_{31}^{2}$ within their allowed range, for a given prior on $\sin ^{2} \theta_{12}$. One sees from the figure that, depending on the $\sin ^{2} \theta_{13}$ error, the octant sensitivity increases or decreases. For example, from the cyan curve for DUNE or T2HK, one sees that the $1 \%$ error corresponds to $5 \sigma$ sensitivity for $\sin ^{2} \theta_{23}$ (true) $<0.45$ and $\sin ^{2} \theta_{23}$ (true) $>0.58$. As the the error increases up to around $6 \%$, the sensitivity is gradually lost. So the measurement of the octant of $\theta_{23}$ strongly depends on the relative error of the $\sin ^{2} \theta_{13}$ determination. The octant discrimination sensitivity is slightly better for T2HK than DUNE due to its high statistics.

In fig. 3, we generated the data assuming $\delta_{\mathrm{CP}}($ true $)=$ $1.45 \pi$. So, it is natural to ask what would be the octant sensitivity over the entire $\sin ^{2} \theta_{23}$ (true) and $\delta_{\mathrm{CP}}$ (true) plane. Fig. 4 provides a clear answer to this question. The simulation details are exactly the same as for fig. 3 . This figure neatly summarizes the effect of the relative $\sin ^{2} \theta_{13}$ error upon the octant sensitivity. We have assumed a $4 \sigma$ confidence level for the exclusion of the wrong octant and then varied the various error combinations as indicated by the different colors. A band of uncertainty clearly arises, decreasing the $4 \sigma$ sensitivity range for $\sin ^{2} \theta_{23}$ (true). It is important to notice that our result is almost independent of $\delta_{\mathrm{CP}}$ (true). As discussed earlier, T2HK gives slightly better sensitivity than DUNE.
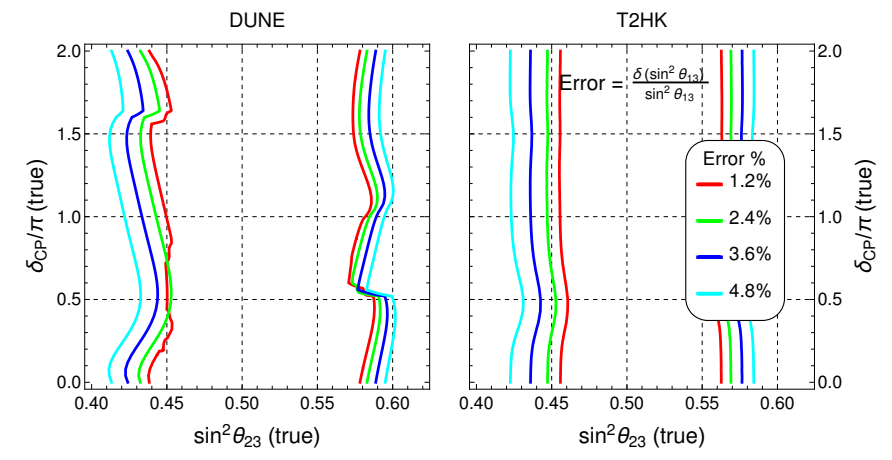

FIG. 4. Octant discrimination potential at $4 \sigma\left(\Delta \chi^{2}=19.33\right)$ confidence level in the $\left[\sin ^{2} \theta_{23}, \delta_{\mathrm{CP}}\right]$ (true) plane. The red, green, blue and cyan curves delimit the "octant-blind" regions corresponding to $1.2 \%, 2.4 \%, 3.6 \%$ and $4.8 \%$ relative errors on $\sin ^{2} \theta_{13}$.

\section{Other T2HK setups}

Here we further elaborate upon the T2HK experimental setup. The details of the T2HK setup for Fig. 1 have been already described in sec. III. But for right panel of Fig. 5 we have considered $295 \mathrm{~km}$ of baseline and two 187 kton tank as Water Cherenkov far detector placed in Japan at an off-axis angle of $2.5^{\circ}$.
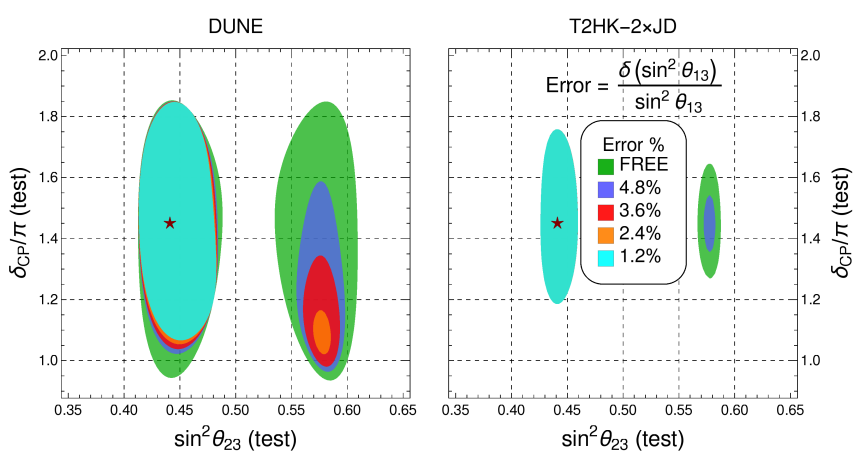

FIG. 5. Measurement of $\theta_{23}$ and $\delta_{C P}$ at $4 \sigma\left(\Delta \chi^{2}=19.33\right)$ confidence. The symbol "star" denotes $\sin ^{2} \theta_{23}^{\text {TRUE }}=0.441$ and $\delta_{\mathrm{CP}}^{\mathrm{TRUE}}=1.45 \pi$. Left (Right) panels correspond to DUNE (T2HK). Differently shaded (colored) regions correspond to various errors associated with $\sin ^{2} \theta_{13}$.

We call this setup as T2HK- $2 \times$ JD. A total exposure of $(1.3 \mathrm{MW}) \times\left(10 \times 10^{7} \mathrm{Sec}\right)$ with a $1: 3 \nu$ and $\bar{\nu}$ running ratio has been assumed. We have assumed an uncorrelated 5\% (3.5\%) signal normalization, $10 \%$ background normalization error, and 5\% energy calibration error for $\nu$ and $\bar{\nu}$ appearance (disappearance) channel. The event numebers and other details have been matched with [40]. From these two figures it is clear that the impact of two 
setups is not significantly different from each other rather they are very similar from the perspective of this work. We have also checked that the result remain also valid for the setup T2HK-JD + KD following the same reference [40], where one detector with 187 kton fiducial mass is placed in Japan having baseline $295 \mathrm{~km}$ and another detector with 187 kton fiducial mass is placed in Korea having baseline $1100 \mathrm{~km}$.

\section{CONCLUSIONS}

Based upon the current global information on neutrino oscillation parameters we have performed a quantitative analysis of the potential of upcoming long baseline experiments DUNE and T2HK in resolving the atmospheric octant ambiguity. We have found that a precise measurement of the reactor angle $\theta_{13}$ plays a key role in resolving the octant of the atmospheric angle $\theta_{23}$ using such future accelerator neutrino experiments. This highlights the complementarity of reactor and accelerator-based studies in gaining fundamental information on neutrino properties. However, in contrast to the determination of the neutrino mass ordering, no matter how good the precision on $\theta_{13}$ reached in future studies, there will always be an octant blind band in any experiment, close to the limit $\theta_{23} \rightarrow \pi / 4$.

\section{ACKNOWLEDGEMENTS}

Work supported by Spanish grants FPA2014-58183-P, Multidark CSD2009-00064, SEV-2014-0398 (MINECO), PROMETEOII/2014/084 (Generalitat Valenciana). P. S. P. acknowledges the support of FAPESP/CAPES grant 2014/05133-1, 2015/16809-9 and 2014/19164-6.
[1] T. Kajita, Rev. Mod. Phys. 88, 030501 (2016).

[2] A. B. McDonald, Rev. Mod. Phys. 88, 030502 (2016).

[3] D. V. Forero, M. Tortola, and J. W. F. Valle, Phys. Rev. D90, 093006 (2014), 1405.7540.

[4] F. Capozzi, E. Lisi, A. Marrone, D. Montanino, and A. Palazzo, Nucl. Phys. B908, 218 (2016), 1601.07777.

[5] I. Esteban, M. C. Gonzalez-Garcia, M. Maltoni, I. Martinez-Soler, and T. Schwetz, JHEP 01, 087 (2017), 1611.01514 .

[6] G. L. Fogli and E. Lisi, Phys. Rev. D54, 3667 (1996), hep-ph/9604415.

[7] H. Minakata, H. Sugiyama, O. Yasuda, K. Inoue, and F. Suekane, Phys. Rev. D68, 033017 (2003), [Erratum: Phys. Rev.D70,059901(2004)], hep-ph/0211111.

[8] M. Maltoni, T. Schwetz, M. A. Tortola, and J. W. F. Valle, New J. Phys. 6, 122 (2004), hep-ph/0405172.

[9] J. W. F. Valle, Phys. Lett. B199, 432 (1987).

[10] O. G. Miranda and J. W. F. Valle, Nucl. Phys. B908, 436 (2016), 1602.00864

[11] O. G. Miranda, M. Tortola, and J. W. F. Valle, Phys. Rev. Lett. 117, 061804 (2016), 1604.05690.

[12] F. J. Escrihuela, D. V. Forero, O. G. Miranda, M. TÃşrtola, and J. W. F. Valle (2016), 1612.07377.

[13] H. Nunokawa, Y. Z. Qian, A. Rossi, and J. W. F. Valle, Phys. Rev. D54, 4356 (1996), hep-ph/9605301.

[14] D. Grasso, H. Nunokawa, and J. W. F. Valle, Phys. Rev. Lett. 81, 2412 (1998), astro-ph/9803002.

[15] L. Wolfenstein, Phys. Rev. D17, 2369 (1978).

[16] T. S. Kosmas, D. K. Papoulias, M. Tortola, and J. W. F. Valle (2017), 1703.00054.
[17] S. K. Agarwalla, S. Prakash, and S. U. Sankar, JHEP 07, 131 (2013), 1301.2574.

[18] S. K. Agarwalla, S. Prakash, and S. Uma Sankar, JHEP 03, 087 (2014), 1304.3251.

[19] A. Chatterjee, P. Ghoshal, S. Goswami, and S. K. Raut, JHEP 06, 010 (2013), 1302.1370.

[20] M. Bass et al., Phys. Rev. D91, 052015 (2015), 1311.0212 .

[21] K. Bora, D. Dutta, and P. Ghoshal, Mod. Phys. Lett. A30, 1550066 (2015), 1405.7482.

[22] C. R. Das, J. Maalampi, J. Pulido, and S. Vihonen, JHEP 02, 048 (2015), 1411.2829.

[23] N. Nath, M. Ghosh, and S. Goswami, Nucl. Phys. B913, 381 (2016), 1511.07496.

[24] D. Dutta, P. Ghoshal, and S. K. Sehrawat (2016), 1610.07203.

[25] S. K. Agarwalla, S. S. Chatterjee, and A. Palazzo, Phys. Lett. B762, 64 (2016), 1607.01745.

[26] S. K. Agarwalla, S. S. Chatterjee, and A. Palazzo, Phys. Rev. Lett. 118, 031804 (2017), 1605.04299.

[27] R. Acciarri et al. (DUNE) (2015), 1512.06148.

[28] K. Abe et al. (Hyper-Kamiokande Proto-Collaboration), PTEP 2015, $053 \mathrm{C} 02$ (2015), 1502.05199.

[29] E. K. Akhmedov, R. Johansson, M. Lindner, T. Ohlsson, and T. Schwetz, JHEP 04, 078 (2004), hep-ph/0402175.

[30] P. A. N. Machado, H. Minakata, H. Nunokawa, and R. Zukanovich Funchal, JHEP 05, 109 (2014), 1307.3248.

[31] S. S. Chatterjee, P. Pasquini, and J. W. F. Valle (2017), 1702.03160

[32] P. Huber, M. Lindner, and W. Winter, Comput. Phys. Commun. 167, 195 (2005), hep-ph/0407333. 
[33] P. Huber, J. Kopp, M. Lindner, M. Rolinec, and W. Winter, Comput. Phys. Commun. 177, 432 (2007), hep$\mathrm{ph} / 0701187$.

[34] A. Cabrera, Double chooz (new multi-detector results), ep seminar, indico.cern.ch/event/548805/attachments/ 1336343/2017513/DCIVCERN_REF_Anatael_16Sept.pdf

[35] J. H. Choi et al. (RENO), Phys. Rev. Lett. 116, 211801 (2016), 1511.05849.

[36] F. P. An et al. (Daya Bay) (2016), 1610.04802.

[37] S.-H. Seo, in Neutrino Oscillation Workshop (NOW 2016): International Workshop on Neutrino and Astroparticle Physics (NOW 2016) Otranto (Lecce), Italy,
September 4-11, 2016 (2017), 1701.06843, URLhttps: //inspirehep.net/record/1510440/files/arXiv: 1701.06843.pdf

[38] A. M. Dziewonski and D. L. Anderson, Physics of the Earth and Planetary Interiors 25, 297 (1981), ISSN 0031-9201, URLhttp://www.sciencedirect.com/ science/article/pii/0031920181900467

[39] F. D. Stacey, Physics of the earth, vol. 2nd ed. (Wiley, 1977).

[40] K. Abe et al. (Hyper-Kamiokande proto-) (2016), 1611.06118 . 